journal club

\title{
Welche diagnostische Methode hat die höchste Trefferquote?
}

Fragestellung: Welches bildgebende diagnostische Verfahren (CT-Angiografie, Kernspinangiografie oder digitale Subtraktionsangiografie) hat die höchste Trefferquote, um bei intrazerebralen Blutungen makrovaskuläre Ursachen zu identifizieren?

Hintergrund: Bei $10-15 \%$ aller Schlaganfälle handelt es sich um intrazerebrale Blutungen. Zu den makrovaskulären Ursachen intrazerebraler Blutungen zählen arteriovenöse Malformationen, Aneurysmen, arteriovenöse Fisteln, Kavernome und zerebrale Sinusvenenthrombosen. Die meisten dieser makrovaskulären Blutungsursachen haben therapeutische Konsequenzen. Daher sind der bildgebende Nachweis beziehungsweise die Identifizierung der zugrunde liegenden Ursache sehr wichtig. Zur Bildgebung stehen die CT-Angiografie, die Kernspinangiografie und die digitale Subtraktionsangiografie zur Verfügung.

Patienten und Methodik: Die prospektive multizentrische DIAGRAM-Studie (Diagnostik AngioGRAphy to find vascular Malformations) schloss Patienten mit nicht traumatischen intrazerebralen Blutungen im Alter zwischen 18 und 70 Jahren ein, die in 22 Krankenhäusern in den Niederlanden zwischen Juli 2008 und Juni 2014 behandelt wurden.

Patienten mit Blutungen in den Basalganglien, im Thalamus und in der hinteren Schädelgrube waren von der Studienteilnehme ausgeschlossen. Darüber hinaus wurden Patienten ausgeschlossen, bei denen die intrazerebrale Blutung sicher auf eine Hypertonie zurückgeführt werden konnte.

In die Studie wurden ins-

van Asch CJ, Velthuis BK, Rinkel GJ et al; DIAGRAM Investigators. Diagnostic yield and accuracy of $\mathrm{CT}$ angiography, MR angiography, and digital subtraction angiography for detection of macrovascular causes of intracerebral haemorrhage: prospective, multicentre cohort study. BMJ 2015; 351: h5762 gesamt 298 Patienten rekrutiert. Die CT-Angiografie wurde bei allen Patienten innerhalb von sieben Tagen nach der Blutung durchgeführt. Wenn die CT-Angiografie unauffällig war, erfolgte nach Ablauf von vier bis acht Wochen eine Kernspintomografie mit Kernspinan- giografie. Wenn beide Schnittbildverfahren negativ waren, wurde eine digitale Subtraktionsangiografie durchgeführt. Die Beurteilung der Bildgebung erfolgte durch drei Neuroradiologen, die verblindet waren.

Ergebnisse: Bei 69 Patienten, dies entspricht $23 \%$ der Studienteilnehmer, wurde eine makrovaskuläre Ursache der intrazerebralen Blutung festgestellt. In abnehmender Reihenfolge waren dies arteriovenöse Malformationen, durale arteriovenöse Fisteln, Kavernome, ein Aneurysma, eine zerebrale Sinusvenenthrombose und eine angeborene venöse Anomalie.

Bei $98 \%$ der Patienten $(n=291)$ erfolgte eine CT-Angiografie. Bei 214 Patienten mit normaler CT und CT-Angiografie wurden eine zusätzliche Kernspintomografie und Kernspinangiografie durchgeführt. Bei 97 Patienten war der Untersuchungsbefund negativ. Bei diesen 97 Patienten wurde dann zur weiteren Diagnostik eine digitale Subtraktionsangiografie durchgeführt.

Die initiale CT-Angiografie identifizierte 51 makrovaskuläre Blutungsursachen mit einer diagnostischen Trefferquote von $17 \%$. Die Kombination von CT und Kernspintomografie identifizierte zwei zusätzliche makrovaskuläre Ursachen. Die digitale Subtraktionsangiografie fand weitere 15 Ursachen. Erwartungsgemäß wurden Kavernome nur mithilfe der Kernspintomografie entdeckt.

Der positive prädiktive Wert der CT-Angiografie betrug $72 \%$, der zusätzlichen Kernspintomografie und Kernspinangiografie $95 \%$ und der digitalen Subtraktionsangiografie $100 \%$.

Schlussfolgerungen: Die Autoren kommen zu dem Schluss, dass die CT-Angiografie als initiale diagnostische Maßnahme zur Identifikation makrovaskulärer Blutungsursachen bei Patienten mit intrazerebralen Blutungen geeignet ist. Die Trefferquote ist allerdings gering, sodass bei Patienten mit normaler CT-Angiografie eine digitale Subtraktionsangiografie erfolgen sollte. Kavernome können nur mithilfe der Kernspintomografie identifiziert werden.

\section{Befriedigende diagnostische Treffsicherheit der CT-Angiografie}

Diese an 22 Krankenhäusern in den Niederlanden durchgeführte prospektive Kohortenstudie zeigt eine befriedigende diagnostische Treffsicherheit einer initialen CT-Angiografie bei Patienten mit nicht hypertensiv und nicht traumatisch bedingten intrazerebralen Blutungen. Im klinischen Alltag würde man in Deutschland allerdings nicht vier bis acht Wochen warten, bis dann eine Kernspinangiografie oder eine digitale Subtraktionsangiografie durchgeführt wird. Angesichts der geringen diagnostischen Ausbeute der Kernspinangiografie würde man bei normaler CT-Angiografie in der Regel eine digitale Subtraktionsangiografie durchführen. 